\title{
Effects of oxygen concentration on the proliferation and differentiation of mouse neural stem cells in vitro
}

Short running title: Effects of oxygen concentration on neural stem cells

Nobutaka Horie, MD, PhD; Kenji So, MD; Takahiro Moriya, PhD; Naoki Kitagawa, MD, Keisuke Tsutsumi, MD., Izumi Nagata, MD, PhD; Kazuyuki Shinohara, MD, PhD

Department of Neurobiology and Behavior, Unit of Basic Medical Sciences, Nagasaki University Graduate School of Biomedical Sciences (N.H., T.M., K.S., K.S.); Department of Neurosurgery, Nagasaki University Graduate School of Medicine, Nagasaki (N.H., N.K, K.T., I.N.); Japan

Corresponding author: Kazuyuki Shinohara (kazuyuki@nagasaki-u.ac.jp) and Nobutaka Horie (nhorienagasaki@yahoo.co.jp), 1-12-4 Sakamoto, Nagasaki, 852-8523, Japan, E-mail address: Tel: +81-95-849-7033, Fax: +81-95-849-7036 


\begin{abstract}
Background and purpose- Cerebral ischemia is known to elicit the activation of neural stem cells (NSCs), however its mechanism is not fully determined. Although oxygen concentration is known to mediate many ischemic actions, there has been little attention given to the role of pathological oxygen changes under cerebral ischemia on the activation of NSCs. We investigated the effects of various oxygen concentrations on mouse neural stem cells in vitro.
\end{abstract}

Methods- NSCs were cultured from the ganglionic eminence of fetal ICR mice on embryonic day 15.5 using a neurosphere method. The effects of oxygen concentrations on proliferation, differentiation and cell death of NSCs were evaluated by bromodeoxyuridine (BrdU) incorporation, immunocytochemistry and TUNEL assay, respectively.

Results- The highest proliferation and the neuronal differentiation of the NSCs were observed in $2 \%$ oxygen, which yielded significantly higher proportions of both BrdU-labeled cells and Tuj1-positive cells when compared with $20 \%$ and $4 \%$ oxygen. On the other hand, the differentiation to the astrocytes was not affected by oxygen concentrations, except in the case of anoxia ( $0 \%$ oxygen). The cell death of the NSCs increased in lower oxygen conditions and peaked at anoxia. Furthermore, the switching of the neuronal subtype differentiation from GABA-positive to glutamate-positive neurons was observed in lower oxygen conditions.

Conclusions- These findings raise the possibility that reduced oxygen levels occurring with cerebral ischemia enhances NSC proliferation and neural differentiation, and that mild hypoxia (2 \% oxygen), which is known to occur in the ischemic penumbra, is suitable for abundant neuronal differentiation.

Key Words: neural stem cell, hypoxia, proliferation, differentiation, cerebral ischemia 


\section{Introduction}

Cerebral ischemia is known to cause a complex cascade of cellular events, leading to both acute and delayed neural death and severe brain dysfunction in animal models as well as in human beings (Siesjo 1992). Interestingly, a recent line of evidence demonstrates that global and focal ischemia enhances the proliferation and the neural differentiation of neural stem cells (NSCs) in the subgranular zone of the dentate gyrus, the anterior subventricular zone and the posterior periventriclar zone adjacent to the hippocampus. These conditions also increase the migration of the newborn neurons to the dentate granule cell layer, the olfactory bulb, the striatum, and the damaged hippocampal CA1 region (Arvidsson et al. 2002; Kee et al. 2001; Liu et al. 1998; Nakatomi et al. 2002; Sato et al. 2001; Takagi et al. 1999; Yagita et al. 2001). However, the mechanisms underlying the activation of the NSCs (enhanced proliferation, neural differentiation and migration) triggered by ischemia are not fully understood.

Among various incidents during ischemia, reductions in the supply of oxygen (hypoxia) and glucose (hypoglycemia) in the brain seem to be the main factors mediating ischemic actions in the brains. Recently, we have reported that when the NSCs were cultured at lowered concentrations of glucose, which is reported to occur in the brain under physiological and pathophysiological conditions, their proliferation and differentiation into neurons and astrocytes is facilitated in vitro (Horie et al. 2004). With regard to oxygen concentration, it has been reported that physiological oxygen concentrations in the brain (3-5\%) enhances the proliferation of cultured NSCs and modulates their differentiation into neurons. This is in contrast conditions of $20 \%$ oxygen, which is non-physiologically high in vivo, but often used in the standard 
method of cell culture (Jin et al. 2002; Morrison et al. 2000; Shingo et al. 2001; Studer et al. 2000). However, there have not been any studies investigating the effect of lower concentrations of oxygen seen in pathologically conditions on the proliferation and the differentiation of the NSCs. It is well known that acute ischemic stroke lowers the oxygen concentration to various levels depending on the brain area (Astrup et al. 1981; Fisher 1997; Harris et al. 1987; Liu et al. 2004; Studer et al. 2000). In the severely damaged ischemic core regions, blood supply is interrupted and oxygen concentration decreases to anoxia level (near $0 \%$ ). In the penumbra, which surrounds the core region and is initially viable tissue, there is a reduced but constant blood supply, leading to lowered tissue oxygen concentration of approximately $2 \%$, which is approximately half the oxygen concentration in the normal brain tissue (3 to $5 \%$ ) (Harris et al. 1987; Liu et al. 2004; Studer et al. 2000).

Considering the various concentrations of oxygen under physiological and pathophysiological conditions, we examine the effects of oxygen concentrations $(0,1,2$, 3, 4, 10 and $20 \%$ ) on the proliferation and differentiation, as well as, the cell death of the NSCs derived from embryonic mouse ganglion eminence by means of an in vitro NSC culture system. An in vitro system allows us to control oxygen concentrations and delineate the actions of the extrinsic factors.

\section{Materials and Methods}

\section{Primary NSC culture}

The NSCs were isolated and propagated using the neurosphere method developed by Reynolds, Weiss and colleagues (Reynolds et al. 1992; Reynolds and Weiss 1992). 
Fetal ICR mice on embryonic day 15.5 (midnight of overnight mating is designated as embryonic day 0) were isolated from their mother under deep anesthesia with ether and placed into ice-cold 1:1 mixture of Dulbecco's modified Eagle's medium and F-12 nutrient (DMEM/F-12: Gibco-BRL, Gaithersburg, Md., USA). The ganglionic eminence tissue was carefully micro-dissected under a stereomicroscope and triturated with a commercial $1 \mathrm{ml}$ plastic pipette to obtain a single cell suspension in $\mathrm{N}-2$ medium (DMEM/F-12 supplemented with $36.4 \mathrm{mM}$ glucose, $23 \mu \mathrm{g} / \mathrm{ml}$ insulin, $92 \mu \mathrm{g} / \mathrm{ml}$ transferrin, $55 \mu \mathrm{M}$ putrescine, $27.5 \mathrm{nM}$ sodium selenite, $20 \mathrm{nM}$ progesterone and 50 U/ml penicillin-streptomycin) (Mitome et al. 2001). The viable dissociated cells at a density of $25 \times 10^{4}$ cells/5 $\mathrm{ml}$ in N-2 medium containing $20 \mathrm{ng} / \mathrm{ml}$ EGF (Invitrogen Corp., Carlsbad, CA, USA) were seeded into uncoated T25 culture flasks (Becton-Dickinson, Franklin Lakes, NJ) and were maintained in a humidified incubator at $37{ }^{\circ} \mathrm{C}$ with $95 \%$ atmospheric air $/ 5 \% \mathrm{CO}_{2}$. Cultures were incubated for 5 days to form enough primary neurospheres. The cell viability for all the primary cultures examined was over $90 \%$ at the beginning of the experiment (data not shown).

\section{BrdU immunocytochemistry}

The effect of oxygen concentrations on the proliferative activity of NSCs was evaluated by bromodeoxyuridine (BrdU) immunocytochemistry (Studer et al. 2000). The single cell suspensions from primary neurospheres were prepared by centrifugation $(1,200 \mathrm{rpm}$, $5 \mathrm{~min}$ ) followed by mechanical dissociation. They were seeded at a cell density of 2.2 x $10^{4}$ cells/350 $\mu \mathrm{l}$ in $\mathrm{N}-2$ medium containing EGF (20 ng/ml), which had been equilibrated under each oxygen concentration $(0,1,2,3,4,10$ and $20 \%)$, in Lab-Tek ${ }^{\circledR}$ 
Chamber Slide $^{\mathrm{TM}}$ (Nunc, Inc., Naperville, IL, USA). The NSC cultures were placed in humidified portable isolation chambers $\left(\mathrm{CO}_{2}\right.$ multi-gas incubator, APMW-36, ASTEC, and AMP-30D, ASTEC, Japan), flushed continuously with a $\mathrm{N}_{2}$ gas to maintain established $\mathrm{O}_{2}$ concentrations at a constant temperature of $37^{\circ} \mathrm{C}$. The oxygen concentration was monitored with IRMA blood analysis system M3623A (Agilent Technologies, Tokyo, Japan) before the experiment. The analyzed data was calculated taking into consideration the appropriate atmospheric pressure of $1009 \mathrm{hPa}$ (756.8mmHg) and the saturation stem pressure of $62.75 \mathrm{hPa}(47.0 \mathrm{mmHg})$ at $37{ }^{\circ} \mathrm{C}$. These measured values were compatible with the established oxygen concentrations in the camber (data not shown). After $24 \mathrm{hr}$ incubation at various concentrations of oxygen, cells were labeled with a $10 \mu \mathrm{M}$ of BrdU and incubated for an additional $6 \mathrm{hr}$. After the labeling medium was removed by aspiration, cells were fixed in PBS containing $4 \%$ paraformaldehyde for $20 \mathrm{~min}$ at room temperature. To denature nuclear DNA into single-strand term, cells were incubated in $0.02 \%$ pepsin in $0.01 \mathrm{~N}$ $\mathrm{HCl}$ for $20 \mathrm{~min}, 2 \mathrm{~N} \mathrm{HCl}$ for $45 \mathrm{~min}$, and $0.15 \mathrm{M}$ Sodium Borate for $10 \mathrm{~min}$. Cells were washed by PBS three times and incubated overnight with anti-BrdU antibody (1: 50; rat monoclonal antibody, Oxford Biotechnology Ltd, Oxford, UK) in PBS containing $0.1 \%$ Triton $\mathrm{X}-100$ and $3 \%$ normal goat serum at $4{ }^{\circ} \mathrm{C}$.

After removal of the primary antibody solution, cells were washed with PBS three times and were incubated with secondary antibodies (Alexa Fluoro ${ }^{\circledR} 568$ goat anti-rat IgG, 1:200, Molecular Probes Inc., Eugene, OR) and $1.25 \mu \mathrm{g} / \mathrm{ml}$ of Hoechst 33258 for a nuclear counter staining for $2 \mathrm{hr}$ at room temperature under light-shading conditions. After sequential washing with PBS and water, the slides were mounted with Gel/ 
Mount $^{\text {TM }}$ (Biomeda corp, Foster City, CA) and their fluorescent images were viewed with a microscope (Nikon E600 and Olympus DP70, Japan). For the quantitative analysis, we counted numbers of BrdU positive cells in a fixed area (700 x $1000 \mu \mathrm{m})$ at the center of each well under the fluorescent-microscope. The number of BrdU positive cells was counted by an observer blind to the treatment condition and the counts were normalized to the total cell number (Hoechst 33258-stained nucleus).

\section{TUNEL assay}

The effect of oxygen concentrations on the cell death of NSCs was evaluated by TUNEL In Situ Cell Death Detection Kit, Fluorescein (Roche, Penzberg, Germany) (Studer et al. 2000). The single cell suspensions from primary neurospheres were prepared by centrifugation $(1,200 \mathrm{rpm}, 5 \mathrm{~min})$ followed by mechanical dissociation. They were seeded at a cell density of $2.2 \times 10^{4}$ cells/350 $\mu \mathrm{l}$ in $\mathrm{N}-2$ medium containing EGF (20 ng/ml), which had been equilibrated to each oxygen concentration $(0,1,2,3,4$, 10 and $20 \%$ ), in Lab-Tek ${ }^{\circledR}$ Chamber Slide $^{\mathrm{TM}}$ (Nunc, Inc., Naperville, IL, USA). The NSC cultures were placed in humidified portable isolation chambers $\left(\mathrm{CO}_{2}\right.$ multi-gas incubator, APMW-36, ASTEC, and AMP-30D, ASTEC, Japan). After $24 \mathrm{hr}$ incubation at various concentrations of oxygen, cells were fixed with $4 \%$ paraformaldehyde for $20 \mathrm{~min}$ at room temperature. Furthermore the cells were permeabilized with $0.1 \%$ Triton $\mathrm{X}-100$ in $0.1 \%$ sodium citrate for 2 min on ice, and incubated with $100 \mu \mathrm{l}$ TUNEL reaction mixture including $1.25 \mu \mathrm{g} / \mathrm{ml}$ of Hoechst 33258 for $60 \mathrm{~min}$ at $37^{\circ} \mathrm{C}$. After sequential washing with PBS and water, the slides were mounted with Gel/ Mount ${ }^{\mathrm{TM}}$ (Biomeda corp) and their fluorescent images were viewed 
with a microscope (Nikon E600 and Olympus DP70). For the quantitative analysis, we counted the number of TUNEL positive cells in a fixed area $(700 \times 1000 \mu \mathrm{m})$ at the center of each well under the fluorescent-microscope. The number of TUNEL positive cells was counted by an observer blind to the treatment condition and the counts were normalized to the total cell number (Hoechst 33258-stained nucleus).

\section{Immunocytochemistry}

The effect of oxygen concentrations on the differentiation of NSCs into neurons and astrocytes was evaluated by immunocytochemical staining for Tuj-1 and GFAP, respectively after differentiation was induced by a low concentration (1\%) of fetal bovine serum (FBS) (Studer et al. 2000). Neuronal subtypes were also evaluated by immunocytochemical staining for GABA, glutamate, tyrosine hydroxylase (TH) and serotonin. After primary neurospheres were dissociated into the single cell suspensions in $1 \%$ FBS-containing $\mathrm{N}-2$ medium lacking EGF in Lab-Tek ${ }^{\circledR}$ Chamber Slide $^{\mathrm{TM}}$, the NSCs were incubated for 2 days at various concentrations of oxygen $(0,1$, 2, 3, 4, 10 and 20\%) in humidified portable isolation chambers described above. The differentiated cells were fixed in PBS containing $4 \%$ paraformaldehyde for $30 \mathrm{~min}$ at room temperature. Cells were incubated overnight with anti-Tuj-1 antibody (1:200; mouse monoclonal antibody, Sigma), anti-GFAP antibody (1:80; rabbit polyclonal antibody, Sigma), anti-GABA antibody (1:500; rabbit polyclonal antibody, Sigma), anti-Glutamate antibody (1:2000; rabbit polyclonal antibody, Sigma), anti-TH antibody (1:200; rabbit polyclonal antibody, Chemicon) and anti-Serotonin antibody (1:500; rabbit polyclonal antibody, Progen) in PBS containing $0.1 \%$ Triton X-100 and $3 \%$ normal goat serum at $4{ }^{\circ} \mathrm{C}$. After removal of the primary antibody solution, cells were 
washed with PBS three times and incubated with two secondary antibodies (Alexa Fluoro ${ }^{\circledR} 488$ goat anti-mouse IgG, 1:200 and Alexa Fluoro ${ }^{\circledR} 568$ goat anti-rabbit IgG, 1:200, Molecular Probes Inc., Eugene, OR) and $1.25 \mu \mathrm{g} / \mathrm{ml}$ of Hoechst 33258 for a nuclear counter staining for $4 \mathrm{hr}$ at room temperature under light-shading conditions. After sequential washing with PBS and water, the slides were mounted with Gel/ Mount ${ }^{\text {TM }}$ (Biomeda corp, Foster City, CA). For the quantitative analysis, we counted the number of Tuj-1-immunoreactive neurons, GFAP-immunoreactive astrocytes, GABA-immunoreactive neurons, glutamate-immunoreactive neurons, TH-immunoreactive neurons and serotonin-immunoreactive neurons in a fixed area (700 $\mathrm{x} 1000 \mu \mathrm{m})$ at the center of each well under the fluorescent-microscope (Nikon E600 and Olympus DP70). The number of immunoreactive cells was counted by an observer blind to the treatment condition and the counts were normalized to the total cell number (Hoechst 33258-stained nucleus).

\section{Statistical analysis}

Results are shown as the mean \pm S.E.M. Statistical difference was analyzed by One-way ANOVA followed by Fisher's PLSD test. Results were considered to be significant when the p value was less than 0.05 .

\section{Results}

\section{The effects of oxygen concentrations on the proliferation of the NSCs}

When NSCs were treated with BrdU for $6 \mathrm{hr}$ at 20\% concentration of oxygen, the 
proportion of BrdU-labeled cells was $10.3 \pm 0.8 \%$ under our experimental conditions (Fig. 1). When compared to $20 \%$ oxygen concentration, which is usually used in the traditional culture method, $10 \%, 4 \%, 3 \%, 2 \%$ and $1 \%$ oxygen concentrations, but not 0\% oxygen concentration, significantly increased the proportion of BrdU-labeled cells in the NSCs. The highest amount of proliferation was observed at 2\% oxygen (28.3 $1.3 \%)$, which was significantly more than at $4 \%$ oxygen concentration ( $24.3 \pm 0.7 \%)$ (Fig. 1).

\section{The effects of oxygen concentrations on the cell death of the NSCs}

With 20\% oxygen concentration, the proportion of TUNEL-positive cells was 9.1土 1.9\% under our experimental conditions (Fig. 2). The proportion of TUNEL-positive cells increased at lower oxygen concentration in a concentration-dependent manner (Fig. 2). When compared to $20 \%$ oxygen concentration, $3 \%, 2 \%, 1 \%$ and $0 \%$ oxygen concentration significantly increased the cell death of the NSCs (Fig. 2).

\section{The effects of oxygen concentrations on the differentiation of the NSCs}

In the next series of experiments, we examined the effect of oxygen concentrations on the differentiation of NSCs. Two days after EGF withdrawal from N-2 medium and the concurrent addition of $1 \%$ FBS at 20\% oxygen concentration, NSCs yielded abundant GFAP-immunoreactive astrocytes $(29.6 \pm 3.1 \%)$ and a small number of

Tuj-1-immunoreactive neurons (4.8 $\pm 0.5 \%)$ (Fig. 3). However, when NSCs were differentiated at $3 \%, 2 \%$ and $1 \%$ of oxygen concentrations, they produced significantly more Tuj-1-immunoreactive neurons when compared with culturing at 
20\% oxygen concentration (Fig. 3). The highest proportion of Tuj1-positive cells was observed at 2\% oxygen. More interestingly, the tendency for NSCs to differentiate into Tuj-1-immunoreactive neurons was significantly higher in $2 \%$ oxygen concentration than in $4 \%$ oxygen concentration (2 \% oxygen: $15.1 \pm 1.0 \%, 4 \%$ oxygen: 7.9 $\pm 0.4 \%$; $=0.04$ ). Moreover, when compared to $20 \%$ oxygen, much lower oxygen concentration ( $0 \%$ and $1 \%)$ did not affect the proportion of Tuj-1-immunoreactive neurons (3.8 $\pm 0.7 \%$ and $10.9 \pm 2.3 \%$ respectively). On the other hand, there was no difference in GFAP-positive cells between $20 \%$ oxygen and any oxygen concentration examined (Fig. 3). The proportion of GFAP-positive cells significantly decreased in $0 \%$ oxygen when compared to $3 \%$ and $2 \%$ oxygen concentrations (Fig. 3).

We next examined the effects of oxygen concentrations on neuronal subtype differentiation of the NSCs. As shown in Fig. 4 (A) and 4 (B), the proportion of GABA-positive neurons was significantly decreased in lower oxygen concentrations (10\%, $4 \%, 3 \%, 2 \%, 1 \%$ and $0 \%$ ) as compared to the $20 \%$ oxygen concentration. On the other hand, the proportion of glutamate- positive neurons was significantly increased in lower oxygen concentrations (3\%, $2 \%, 1 \%$ and $0 \%$ ) as compared to the 20\% oxygen concentration (Fig. 4 (C, D)). We could not find any TH-positive neurons or serotonin-positive neurons in our experimental condition (data not shown).

\section{Discussion}

In the present study, we demonstrated that oxygen concentration is critical for the proliferation and neural differentiation, as well as, cell death of the NSCs. The proliferation and neuronal differentiation of NSCs was enhanced under lowered oxygen 
conditions, especially at a concentration of $2 \%$, which yielded significantly higher proportions of both BrdU-labeled cells and Tuj1-positive cells when compared with $20 \%$ and $4 \%$ oxygen. Also, the switching of the neuronal subtype differentiation from GABA-positive to glutamate-positive neurons was observed in lowered concentration of oxygen. Furthermore, these hypoxia-induced changes in the NSC functions were observed in the range of physiological and pathophysiological concentrations of oxygen (4 - $0 \%$ ). These findings raise the possibility that hypoxia might mediate the enhancement of proliferation and neural differentiation of the NSCs triggered by cerebral ischemia.

The lowered oxygen-induced enhancement of the NSC activity observed in our present study was consistent with previous reports demonstrating that the growth of neural crest stem cells, neuronal progenitor cells or mesencephalic precursor cells is enhanced in lowered oxygen (3-5\%) when compared with high oxygen concentration (20\%) (Morrison et al. 2000; Shingo et al. 2001; Storch et al. 2001; Studer et al. 2000). Thus, it seem to be common phenomenon that proliferation and neural differentiation of the NSCs or neuronal progenitor cells is higher under physiological oxygen than under non-physiologically high oxygen. However, these previous studies compared the effects of non-physiologically high and physiologically normal oxygen concentrations on NSC activities. In this study, we further examined the effects of much lower concentrations of oxygen ( $0,1,2,3$ and $4 \%)$, which occur physiologically and pathophysiologically in the brains in vivo (Harris et al. 1987; Liu et al. 2004; Studer et al. 2000). As previously described, we demonstrated that the proliferation and neural differentiation of NSCs was highest in the $2 \%$ oxygen condition, which occurs in the penumbra, when compared to the other oxygen concentrations examined in this study including $4 \%$ 
oxygen, which is thought to occur in the normal brain. To our knowledge, this is the first report demonstrating that NSC proliferation and differentiation is enhanced under pathophysiological oxygen conditions occurring in cerebral ischemia. Since an interstitial oxygen concentration is reported to fall into $1-2 \%$ in the ischemic penumbra (Harris et al. 1987; Liu et al. 2004; Studer et al. 2000), ischemia-induced facilitation of neurogenesis in the penumbra may be attributed to the direct actions of lowered oxygen concentration on the activities of the NSC.

In marked contrast to $2 \%$ oxygen, $0 \%$ oxygen failed to affect the proliferation and the neural differentiation of the NSCs, and rather increased the cell death approximately 4 fold when compared with $20 \%$ oxygen concentration. This is different from the previous report suggesting that hypoxia reduced apoptosis of the NSCs derived from mesencephalon (Studer et al. 2000). This difference may be attributed to the regional brain differences in vulnerability against hypoxia/ischemia (Pulsinelli et al. 1982; Smith et al. 1984). Thus, the activation of ganglion-derived NSC induced by lowered oxygen is determined by the balance between proliferation followed by neural differentiation, and cell death. Since an interstitial oxygen concentration is reported to fall to around $0 \%$ in the core region (Harris et al. 1987; Liu et al. 2004; Studer et al. 2000), ischemia-induced decrease in neurogenesis in the core region may be attributed to the direct actions of anoxia on the NSC activities. Ohta and colleagues showed that the number of oligodendrocyte progenitor cells increased in the ischemic penumbra after the ischemic insult, while decreasing in the ischemic core (Ohta et al. 2003). These phenomena observed in vivo supports our hypothesis that the ischemic penumbra environment may enhance NSC proliferation and neuronal differentiation. 
It should be noted that the neuronal subtype differentiated from the NSCs switched from GABA-positive to glutamate-positive neurons under lowered oxygen concentrations. Similar to our finfings, Studer and colleagues also demonstrated that neuronal precursors from E12 rat mesencephalon, which had been expanded in lowered oxygen (3\%), differentiated into more dopaminergic and serotonergic neurons, while the differentiation to GABAergic and glutamatergic neuron was reduced under lowered oxygen conditions (Studer et al. 2000). Morrison and colleagues also reported lowered oxygen concentrations under 3-5\% promotes dopaminergic differentiation from neural crest stem cells. Moreover, these differentiated neurons in low oxygen conditions could also synthesize and release dopamine and norepinephrine (Morrison et al. 2000). Storch and colleagues also reported the dopaminergic differentiation from human mesencephalic neural precursor cells in low oxygen conditions (Storch et al. 2001). These reports suggest that the oxygen reduction from non-physiological high condition to physiologically normal condition drastically enhances neuronal, especially projective neuronal, differentiation such as dopaminergic differentiation of CNS precursors in vitro. In the present study, the NSCs derived from the E15.5 ganglionic eminence failed to differentiate into dopaminergic or serotonergic neurons (Studer et al. 2000). Further study is required to elucidate the mechanisms underlying the hypoxia-induced switching of the neural subtype differentiation.

As described previously, hypoglycemia is another main factor mediating ischemic actions and both hypoxia and hypoglycemia additionally or synergistically contribute to the acute and delayed neural death (Siesjo 1992). Recently, we have shown that lowered glucose concentration suppresses the proliferation and increases the neuronal and astroglial differentiation of cultured NSCs (Horie et al. 2004). Thus, there are 
some differences and some similarities in the effects on the NSC activities between hypoxia and hypoglycemia (Horie et al. 2004). The apparent difference is the effect on the proliferation; an increase following lowered oxygen and contrariwise a decrease following lowered glucose utilizing the same cells (derived from E15.5 ganglionic eminence) and culture conditions held constant. Another difference is that lowered glucose facilitated the differentiation into astrocytes, while lowered oxygen had little effect on it. A similar effect occurring with both lowered oxygen and lowered glucose concentration is the enhancement of the neuronal differentiation from NSCs. Thus, these two energy failures under ischemia have different effects on NSC proliferation and differentiation. The ischemia-induced activation of the endogenous NSCs is caused by the additional and synergic actions of the two main incidents; hypoxia and hypoglycemia. Because it is reported that hypoxia and hypoglycemia lead to distinctly different patterns of the intracellular $\mathrm{pH}$ (Knopfel et al. 1998) and gene expression (Brusselmans et al. 2001), these differences may explain the different actions on the NSC functions.

In conclusion, our study provides evidence that lowered oxygen concentration, known to occur under cerebral ischemia, facilitates NSC proliferation and differentiation, and that pathophysiological oxygen concentration (2\%) in penumbra is found to be optimal for NSC to proliferate and neural differentiate into neurons.

\section{Acknowledgements}

The authors thank Anne-Lise Maag and William Slikker (Department of Neurosurgery, Stanford University) for editing the manuscript. 


\section{References}

Arvidsson A, Collin T, Kirik D, Kokaia Z, Lindvall O. (2002) Neuronal replacement from endogenous precursors in the adult brain after stroke. Nat Med 8:963-970.

Astrup J, Siesjo BK, Symon L. (1981) Thresholds in cerebral ischemia - the ischemic penumbra. Stroke 12:723-725.

Brusselmans K, Bono F, Maxwell P, Dor Y, Dewerchin M, Collen D, Herbert JM, Carmeliet P. (2001) Hypoxia-inducible factor-2alpha (HIF-2alpha) is involved in the apoptotic response to hypoglycemia but not to hypoxia. J Biol Chem 276:39192-39196.

Fisher M. (1997) Characterizing the target of acute stroke therapy. Stroke 28:866-872.

Harris RJ, Richards PG, Symon L, Habib AH, Rosenstein J. (1987) pH, K+, and PO2 of the extracellular space during ischaemia of primate cerebral cortex. J Cereb Blood Flow Metab 7:599-604

Horie N, Moriya T, Mitome M, Kitagawa N, Nagata I, Shinohara K. (2004) Lowered glucose suppressed the proliferation and increased the differentiation of murine neural stem cells in vitro. FEBS Lett 571:237-242

Jin K, Mao XO, Sun Y, Xie L, Jin L, Nishi E, Klagsbrun M, Greenberg DA. (2002) Heparin-binding epidermal growth factor-like growth factor: hypoxia-inducible expression in vitro and stimulation of neurogenesis in vitro and in vivo. $J$ Neurosci 22:5365-5373

Kee NJ, Preston E, Wojtowicz JM. (2001) Enhanced neurogenesis after transient global ischemia in the dentate gyrus of the rat. Exp Brain Res 136:313-320.

Knopfel T, Tozzi A, Pisani A, Calabresi P, Bernardi G. (1998) Hypoxic and hypoglycaemic changes of intracellular $\mathrm{pH}$ in cerebral cortical pyramidal 
neurones. Neuroreport 9:1447-1450

Liu J, Solway K, Messing RO, Sharp FR. (1998) Increased neurogenesis in the dentate gyrus after transient global ischemia in gerbils. J Neurosci 18:7768-7778.

Liu S, Shi H, Liu W, Furuichi T, Timmins GS, Liu KJ. (2004) Interstitial pO2 in ischemic penumbra and core are differentially affected following transient focal cerebral ischemia in rats. J Cereb Blood Flow Metab 24:343-349

Mitome M, Low HP, van den Pol A, Nunnari JJ, Wolf MK, Billings-Gagliardi S, Schwartz WJ. (2001) Towards the reconstruction of central nervous system white matter using neural precursor cells. Brain 124:2147-2161.

Morrison SJ, Csete M, Groves AK, Melega W, Wold B, Anderson DJ. (2000) Culture in reduced levels of oxygen promotes clonogenic sympathoadrenal differentiation by isolated neural crest stem cells. J Neurosci 20:7370-7376.

Nakatomi H, Kuriu T, Okabe S, Yamamoto S, Hatano O, Kawahara N, Tamura A, Kirino T, Nakafuku M. (2002) Regeneration of hippocampal pyramidal neurons after ischemic brain injury by recruitment of endogenous neural progenitors. Cell 110:429-441.

Ohta K, Iwai M, Sato K, Omori N, Nagano I, Shoji M, Abe K. (2003) Dissociative increase of oligodendrocyte progenitor cells between young and aged rats after transient cerebral ischemia. Neurosci Lett 335:159-162

Pulsinelli WA, Brierley JB, Plum F. (1982) Temporal profile of neuronal damage in a model of transient forebrain ischemia. Ann Neurol 11:491-498.

Reynolds BA, Tetzlaff W, Weiss S. (1992) A multipotent EGF-responsive striatal embryonic progenitor cell produces neurons and astrocytes. J Neurosci $12: 4565-4574$ 
Reynolds BA, Weiss S. (1992) Generation of neurons and astrocytes from isolated cells of the adult mammalian central nervous system. Science 255:1707-1710.

Sato K, Hayashi T, Sasaki C, Iwai M, Li F, Manabe Y, Seki T, Abe K. (2001) Temporal and spatial differences of PSA-NCAM expression between young-adult and aged rats in normal and ischemic brains. Brain Res 922:135-139.

Shingo T, Sorokan ST, Shimazaki T, Weiss S. (2001) Erythropoietin regulates the in vitro and in vivo production of neuronal progenitors by mammalian forebrain neural stem cells. J Neurosci 21:9733-9743.

Siesjo BK. (1992) Pathophysiology and treatment of focal cerebral ischemia. Part I: Pathophysiology. J Neurosurg 77:169-184.

Smith ML, Auer RN, Siesjo BK. (1984) The density and distribution of ischemic brain injury in the rat following 2-10 min of forebrain ischemia:319-332.

Storch A, Paul G, Csete M, Boehm BO, Carvey PM, Kupsch A, Schwarz J. (2001) Long-term proliferation and dopaminergic differentiation of human mesencephalic neural precursor cells. Exp Neurol 170:317-325.

Studer L, Csete M, Lee SH, Kabbani N, Walikonis J, Wold B, McKay R. (2000) Enhanced proliferation, survival, and dopaminergic differentiation of CNS precursors in lowered oxygen. $J$ Neurosci 20:7377-7383.

Takagi Y, Nozaki K, Takahashi J, Yodoi J, Ishikawa M, Hashimoto N. (1999) Proliferation of neuronal precursor cells in the dentate gyrus is accelerated after transient forebrain ischemia in mice. Brain Res 831:283-287.

Yagita Y, Kitagawa K, Ohtsuki T, Takasawa K, Miyata T, Okano H, Hori M, Matsumoto M. (2001) Neurogenesis by progenitor cells in the ischemic adult rat hippocampus. Stroke 32:1890-1896. 


\section{Figure legends}

Fig.1. The effects of oxygen concentrations on the proliferation of cultured NSCs. NSCs were treated with BrdU $(10 \mu \mathrm{M})$ for $6 \mathrm{hr}$ under various oxygen concentrations and were processed for BrdU immunocytochemistry. The representative immunofluorescence images (A) and the proportions of BrdU-labeled cells (B) are shown. Arrows in (A) indicated the BrdU-labeled cells. When compared to $20 \%$ oxygen, $10 \%, 4 \%, 3 \%, 2 \%$ and $1 \%$, but not $0 \%$ oxygen, significantly increased EGF-induced proliferation. The highest proliferation was observed at $2 \%$ oxygen. The asterisks indicate significant difference compared to $20 \%(p<0.05$; One-way ANOVA followed by Fisher's PLSD test). $n=8$

Fig.2. The effects of oxygen concentrations on cell death of the cultured NSCs. After incubation under various oxygen concentrations, NSCs were processed with the TUNEL assay and the representative immunofluorescence images (A) and the proportions of dead cells (B) are shown. Arrows in (A) indicated the TUNEL-positive cells. The low oxygen culture significantly increased the proportion of dead cells. The asterisks indicate significant difference compared to $20 \%(p<0.05$; One-way ANOVA followed by Fisher's PLSD test). $\quad \mathrm{n}=8$

Fig.3. The effects of oxygen concentrations on the differentiation of cultured NSCs. After the NSCs were differentiated under various oxygen concentrations, they were processed for immunocytochemistry against a neuronal marker, Tuj1 and an astrocyte marker, GFAP, and the representative immunofluorescence images (A) and the 
proportion of Tuj1- (B) and GFAP- (C) positive cells are shown. When compared to $20 \%$ oxygen, $3 \%, 2 \%$ and $1 \%$ significantly increased the proportion of Tuj1-positive cells. The highest proportion of Tuj1-positive cells was observed at $2 \%$ oxygen. In contrast, the proportion of GFAP-positive cells significantly decreased in $0 \%$ oxygen when compared to $3 \%$ and $2 \%$ oxygen concentrations. The asterisks indicate significant difference compared to $20 \%(p<0.05$; One-way ANOVA followed by Fisher's PLSD test). $\quad \mathrm{n}=6$

Fig.4. The effects of oxygen concentrations on neuronal subtype differentiation of the cultured NSCs. After the NSCs were differentiated under various oxygen concentrations, they were processed for immunocytochemistry against a neuronal marker, Tuj1 and GABA (A, B) or glutamate (C, D). The representative immunofluorescence images of Tuj1 and GABA (A) and of Tuj1 and glutamate (C) are shown. The proportion of GABA-positive cells (B) and glutamate positive cells (D) against the number of Tuj1-positice cells are also shown. Arrows in (A) and (C) indicated the double-positive cells. The proportion of GABA-positive neurons was significantly decreased in lower oxygen concentrations, whereas the proportion of glutamate-positive neurons was significantly increased in lower oxygen concentrations. The asterisks indicate significant difference compared to $20 \%(p<0.05$; One-way ANOVA followed by Fisher's PLSD test). $\mathrm{n}=8$ 
(A)

Oxygen concentration (\%)
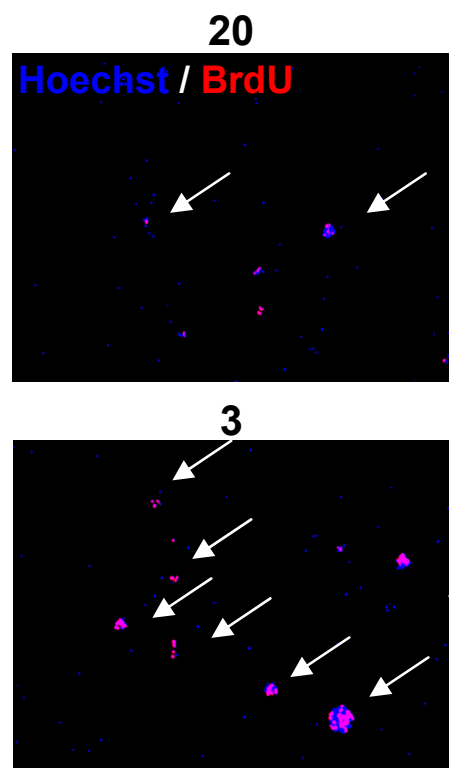

0

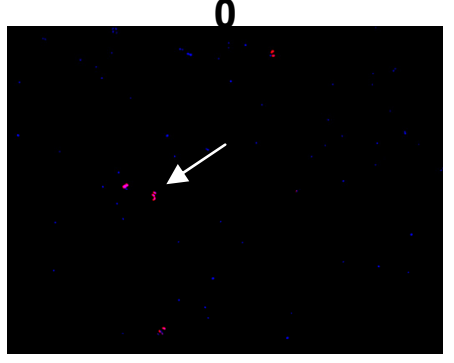

$200 \mu \mathrm{m}$

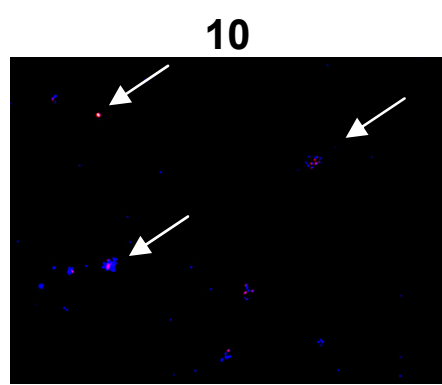

2

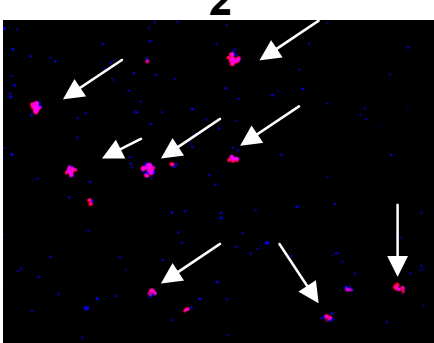

4

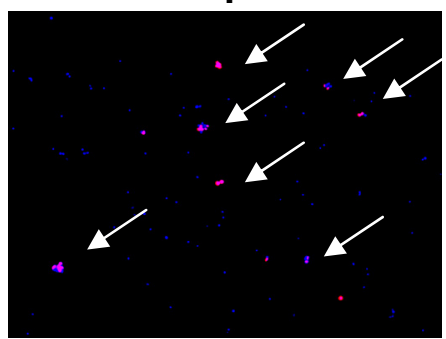

1

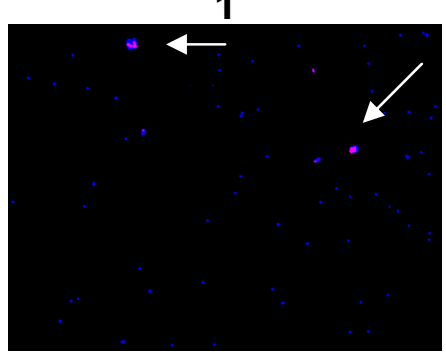

(B)

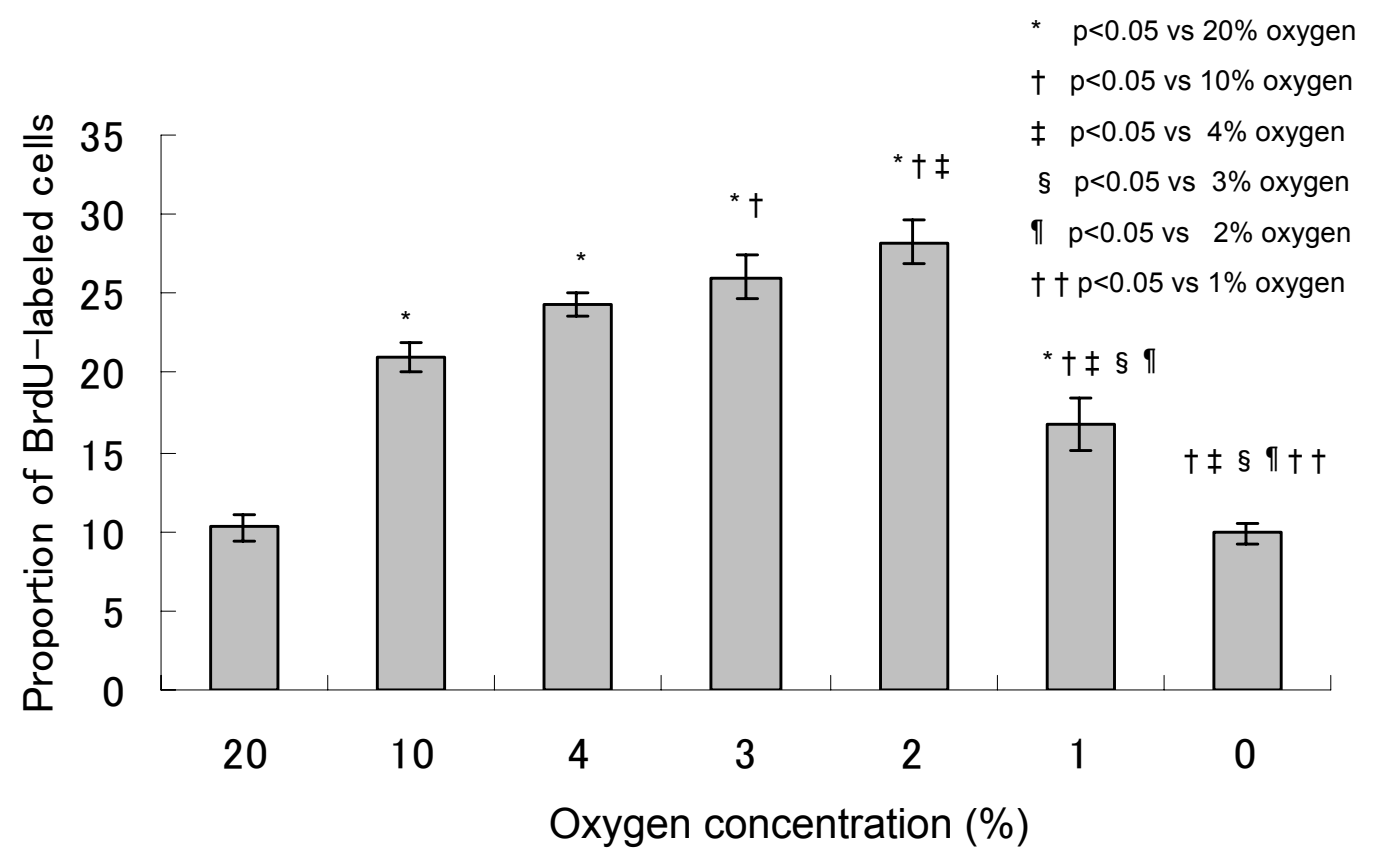

Fig. 1 
(A)

Oxygen concentration (\%)
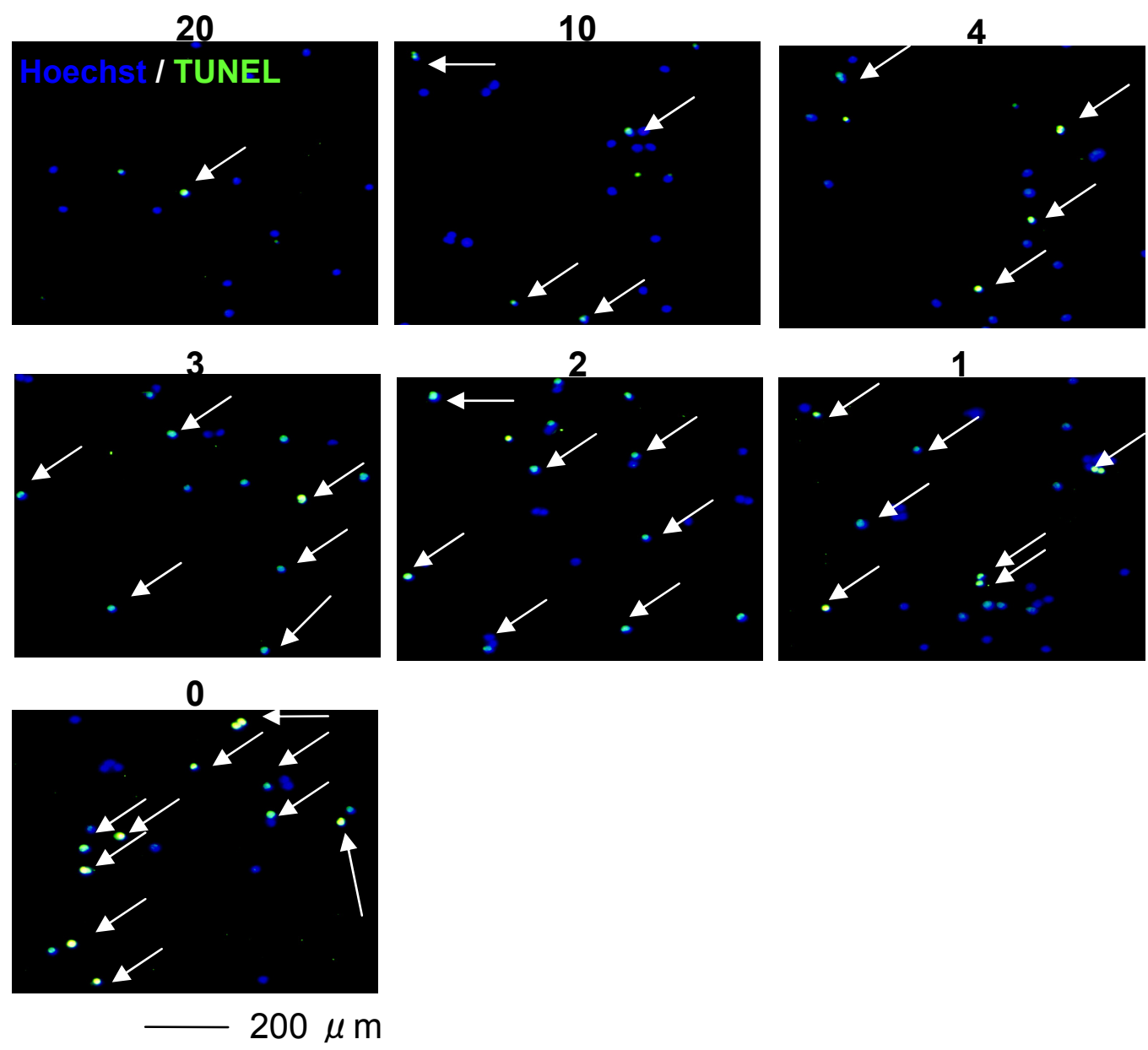

(B)

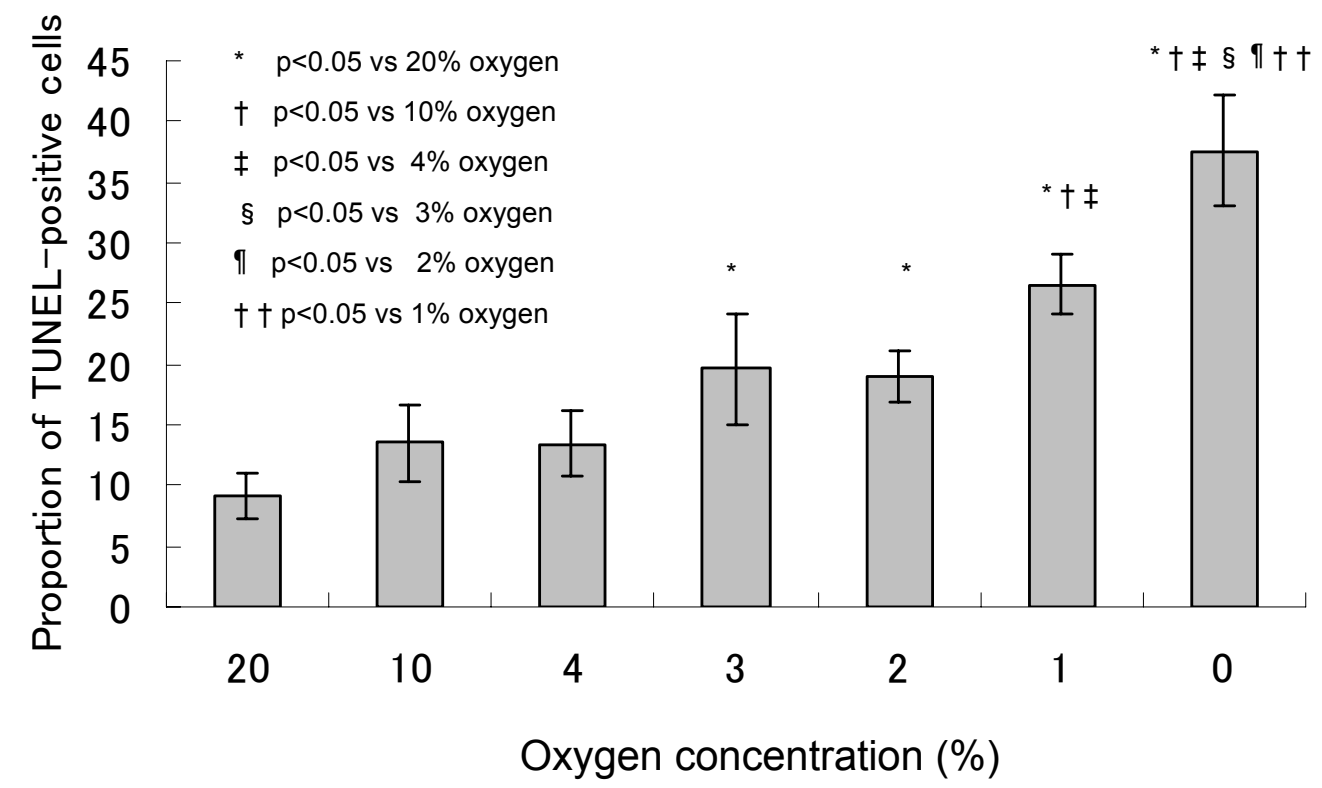

Fig. 2 
(A)

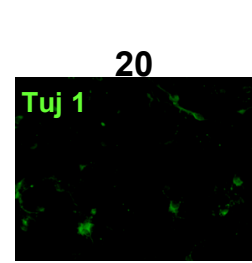

Oxygen concentration (\%)
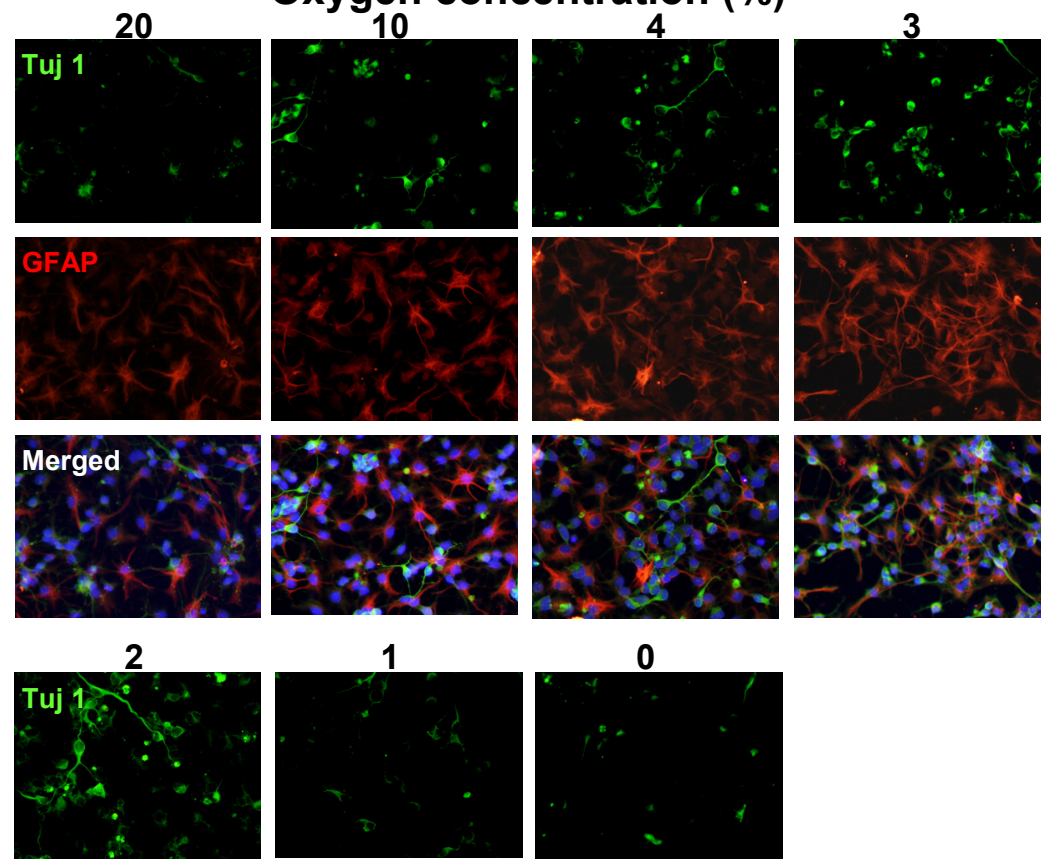

(B)
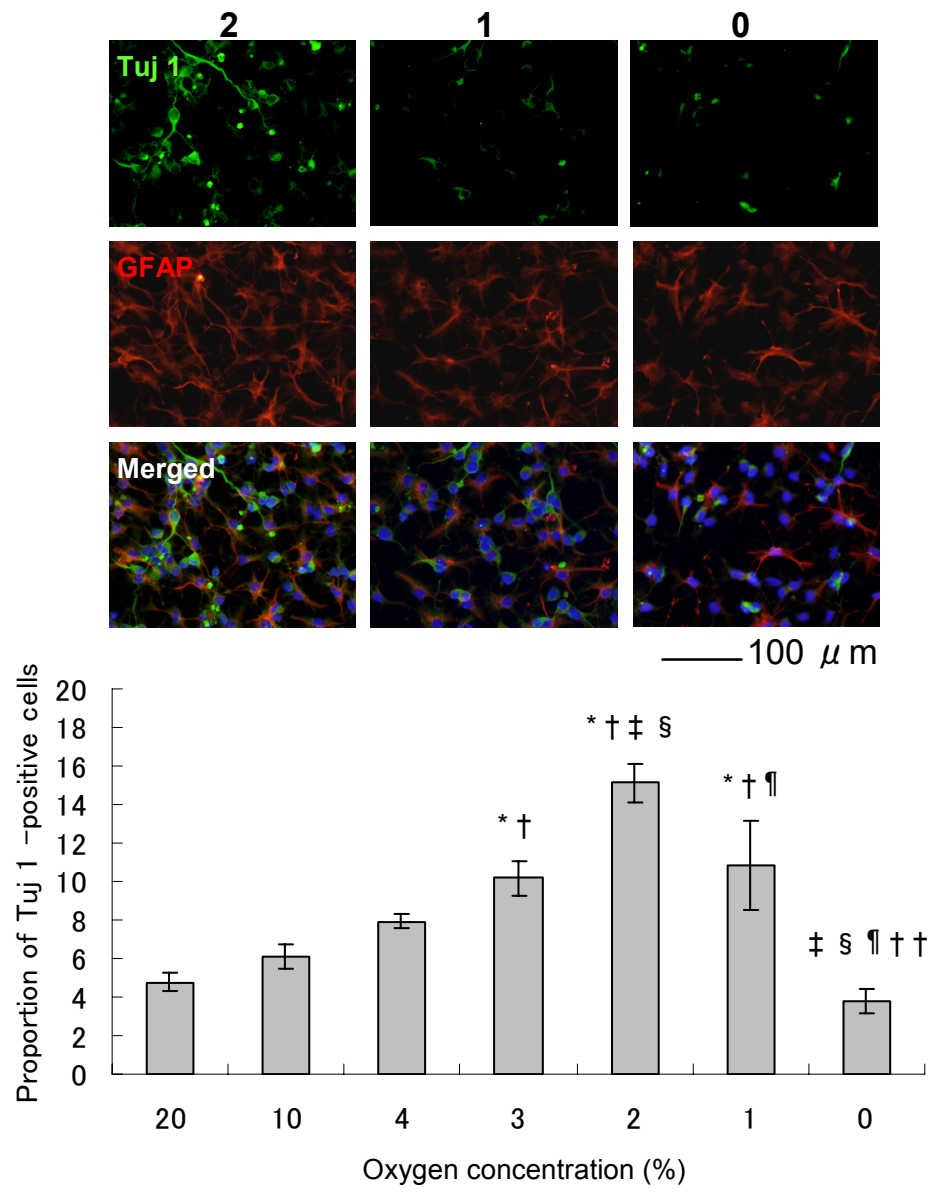

(C)

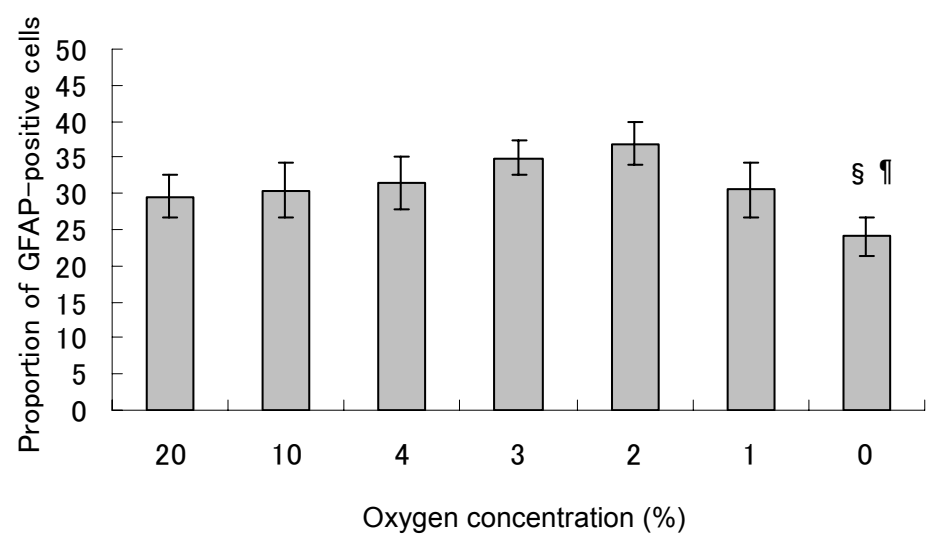

* $p<0.05$ vs $20 \%$ oxygen † $p<0.05$ vs $10 \%$ oxygen $\ddagger p<0.05$ vs $4 \%$ oxygen § $p<0.05$ vs $3 \%$ oxygen If $p<0.05$ vs $2 \%$ oxygen $\dagger+p<0.05$ vs $1 \%$ oxygen 
(A)

\section{Oxygen concentration (\%)}
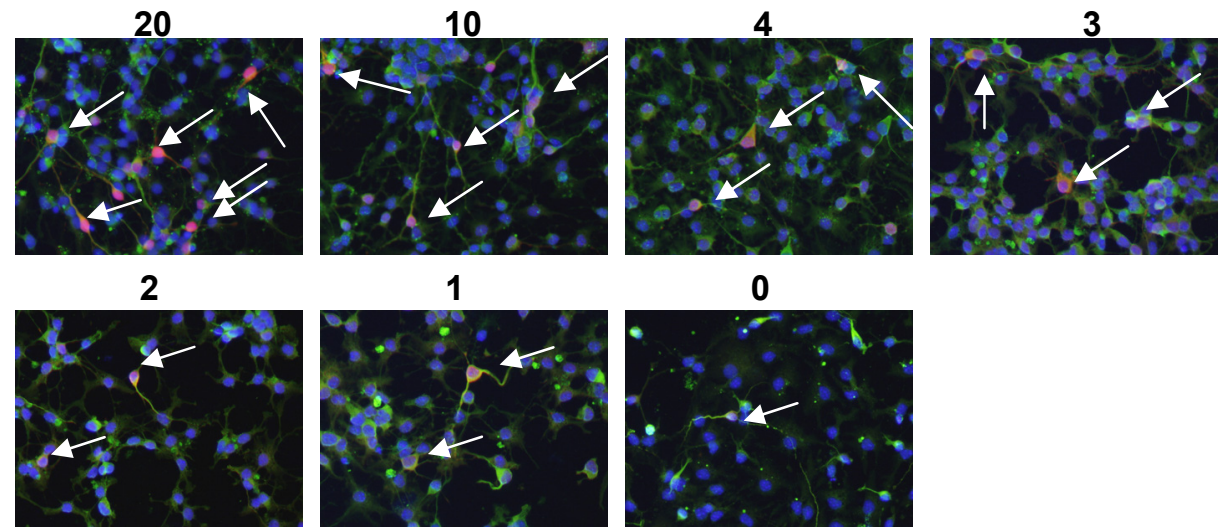

(B)

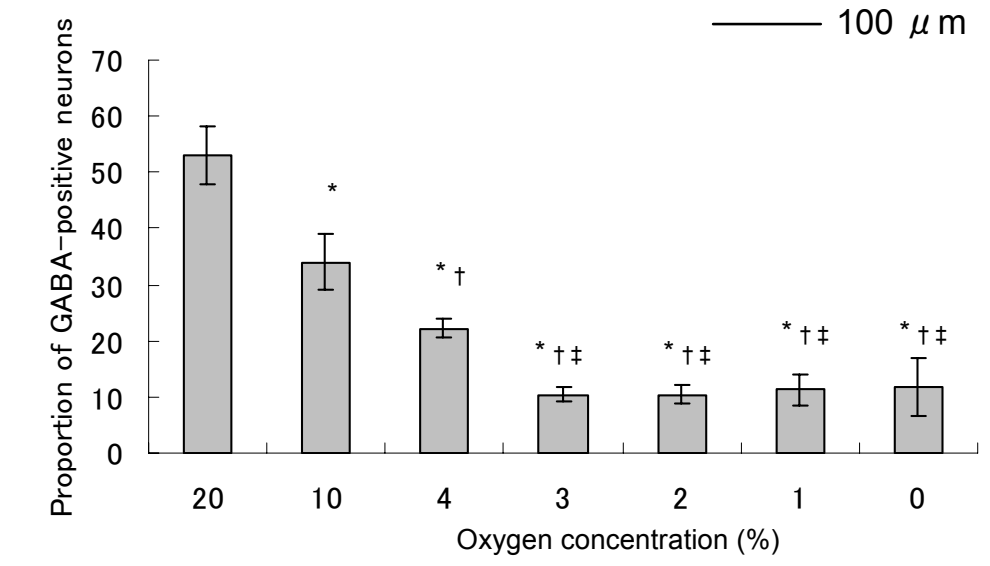

(C)

* $p<0.05$ vs $20 \%$ oxygen

$\dagger p<0.05$ vs $10 \%$ oxygen

$\ddagger p<0.05$ vs $4 \%$ oxygen

$\S p<0.05$ vs $3 \%$ oxygen

If $p<0.05$ vs $2 \%$ oxygen

$\dagger+p<0.05$ vs $1 \%$ oxygen

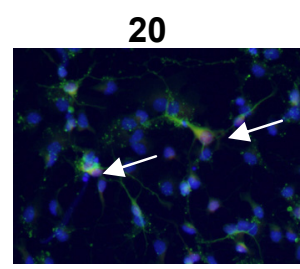

Oxygen concentration (\%)
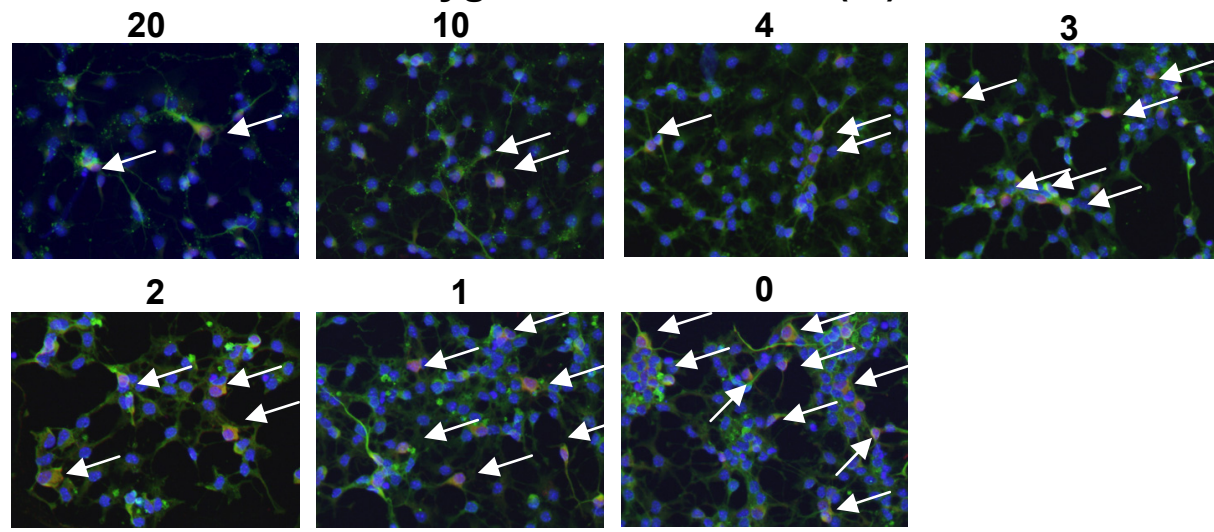

1

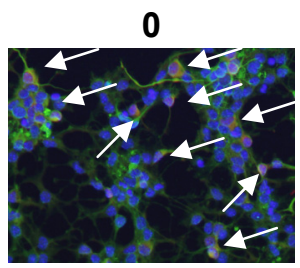

(D)

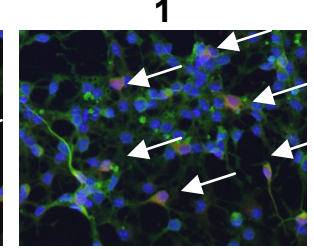

$100 \mu \mathrm{m}$

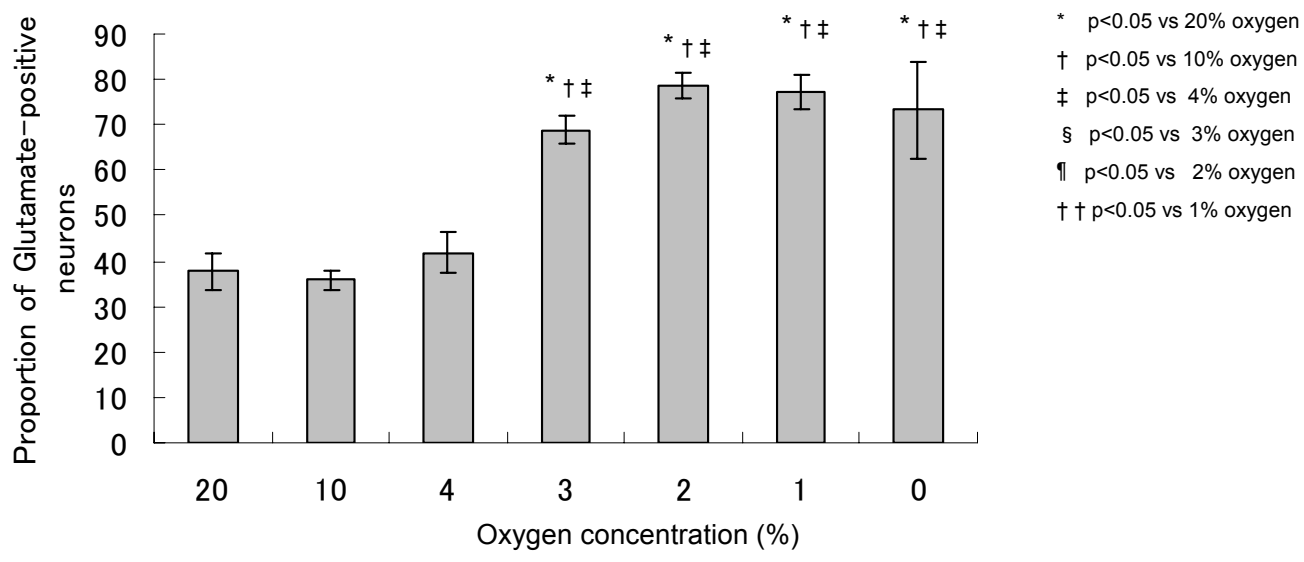

Fig. 4 